\title{
On Entropy of Graph Maps That Give Hereditarily Indecomposable Inverse Limits
}

\author{
Jan P. Boroński ${ }^{1,2}$. Piotr Oprocha ${ }^{2}$
}

Received: 12 January 2015 / Revised: 18 May 2015 / Published online: 5 June 2015

(C) The Author(s) 2015. This article is published with open access at Springerlink.com

\begin{abstract}
We prove that if $f: G \rightarrow G$ is a map on a topological graph $G$ such that the inverse $\operatorname{limit} \lim (G, f)$ is hereditarily indecomposable, and entropy of $f$ is positive, then there exists an entropy set with infinite topological entropy. When $G$ is the circle and the degree of $f$ is positive then the entropy is always infinite and the rotation set of $f$ is nondegenerate. This shows that the Anosov-Katok type constructions of the pseudo-circle as a minimal set in volume-preserving smooth dynamical systems, or in complex dynamics, obtained previously by Handel, Herman and Chéritat cannot be modeled on inverse limits. This also extends a previous result of Mouron who proved that if $G=[0,1]$, then $h(f) \in\{0, \infty\}$, and combined with a result of Ito shows that certain dynamical systems on compact finitedimensional Riemannian manifolds must either have zero entropy on their invariant sets or be non-differentiable.
\end{abstract}

Keywords Topological entropy · Attractor - Hereditarily indecomposable continuum • Pseudo-circle

Mathematics Subject Classification 37B40 $\cdot 37 \mathrm{~B} 45 \cdot 37 \mathrm{C} 70$

\section{Introduction}

It is well known that hereditary indecomposability can be found in the structure of invariant sets of even very regular dynamical systems. Recall that a nondegenerate compact and con-

Piotr Oprocha

oprocha@agh.edu.pl

Jan P. Boroński

jan.boronski@osu.cz

1 National Supercomputing Centre IT4Innovations, Division of the University of Ostrava, Institute for Research and Applications of Fuzzy Modeling, 30. Dubna 22, 70103 Ostrava, Czech Republic

2 Faculty of Applied Mathematics, AGH University of Science and Technology, Al. Mickiewicza 30, 30-059 Kraków, Poland 
nected set, continuum, is said to be indecomposable if it cannot be decomposed to the union of two proper subcontinua, and it is hereditarily indecomposable if every subcontinuum is indecomposable. Handel [26] showed that Bing's pseudo-circle [11], a hereditarily indecomposable cofrontier, can appear as a minimal set for a $C^{\infty}$-smooth volume preserving planar diffeomorphism. A related example was given by Herman [28] in the complex plane. Quite recently Herman's construction has been extended by Chéritat [21] to show that the pseudocircle can also emerge as the boundary of a Siegel disk. Other prominent examples include those given by Kennedy and Yorke, who exhibited that there exist $C^{\infty}$-smooth dynamical systems in dimensions greater than 2 with uncountably many minimal pseudo-circles, and any small $C^{1}$ perturbation of which manifests the same property (see [33,34]). Curiously, it is still an open question whether hereditarily indecomposable continua admit dynamical systems with finite, nonzero entropy (see e.g. [41]). Since by a result of Ito ([31], Theorem 1) topological entropy of a diffeomorphism on a compact finite-dimensional Riemannian manifold must be finite, a potential solution could carry important new insights on the interplay between complexity of dynamics and topology of invariant sets. An important facet of this problem appears to be to determine the answer for shift homeomorphisms on inverse limits of graphs. On the one hand side, inverse limits of graphs are often used to construct attractors of dynamical systems on manifolds, where the dynamics on the attractor is conjugate to the shift homeomorphism (see e.g. $[4,6,7,19,38,43]$ ). On the other hand, famously there are situations where the converse is true: a dynamical system on a manifold can be locally translated to the dynamics of a shift homeomorphism on an inverse system of graphs (see e.g. $[9,14,48])$. Note that, it is well known that many 1-dimensional hereditarily indecomposable continua, such as the pseudo-arc, pseudo-circle or pseudo-solenoids, can be obtained as inverse limits of graphs with a single bonding map (see [32]), and any such bonding map induces a shift homeomorphism on the inverse limit space (see Sect. 3) that, according to [8], extends to a homeomorphism of $\mathbb{R}^{3}$. In the case of the pseudo-arc, techniques to construct appropriate bonding maps on the unit interval were first developed by Henderson [27] (a nontransitive map with zero entropy) and Minc and Transue [37] (a transitive map, hence with positive entropy). It had been known for a while that a bonding map that generates the pseudo-arc as the inverse limit must have entropy at least $\log (2) / 2$, when positive [16], and recently it has been proved by Mouron [42] that such a positive entropy must be in fact infinite. The main ingredient of Mouron's proof is the notion of stretching and taut cover, especially properties of taut covers (and patterns) of hereditarily indecomposable compacta [44]. In this paper we extend Mouron's result and show that if the entropy is positive then there exists an entropy set with infinite entropy. Our approach is not restricted to interval and works for all inverse limits of topological graphs. Our result is obtained by a careful analysis of the structure of horseshoes in graph maps. The main ingredient of our proof is an old result of Brown [18] which associates a degree of "crookedness" to higher iterates of maps which define hereditarily indecomposable compacta as their inverse limits. Combined with a result of Ito, it also shows that if the attractor constructed this way in a compact finite-dimensional Riemannian manifold is hereditarily indecomposable, then the dynamical system must either have zero entropy, or be non-differentiable. In Sect. 4 we discuss an application of our results to hereditarily indecomposable circle-like continua. We show that when $G$ is the circle and the degree of $f$ is positive then the entropy is always infinite and the rotation set of $f$ is nondegenerate. This shows that the Anosov-Katok type constructions of the pseudo-circle as a minimal set in volume-preserving smooth dynamical systems, or in complex dynamics, obtained previously by Handel [26], Herman [28] and Chéritat [21] cannot be modeled on inverse limits. This resembles a known fact for Hénon-type attractors: Williams [48] showed that every hyperbolic, one-dimensional, expanding attractor for a discrete dynamical system 

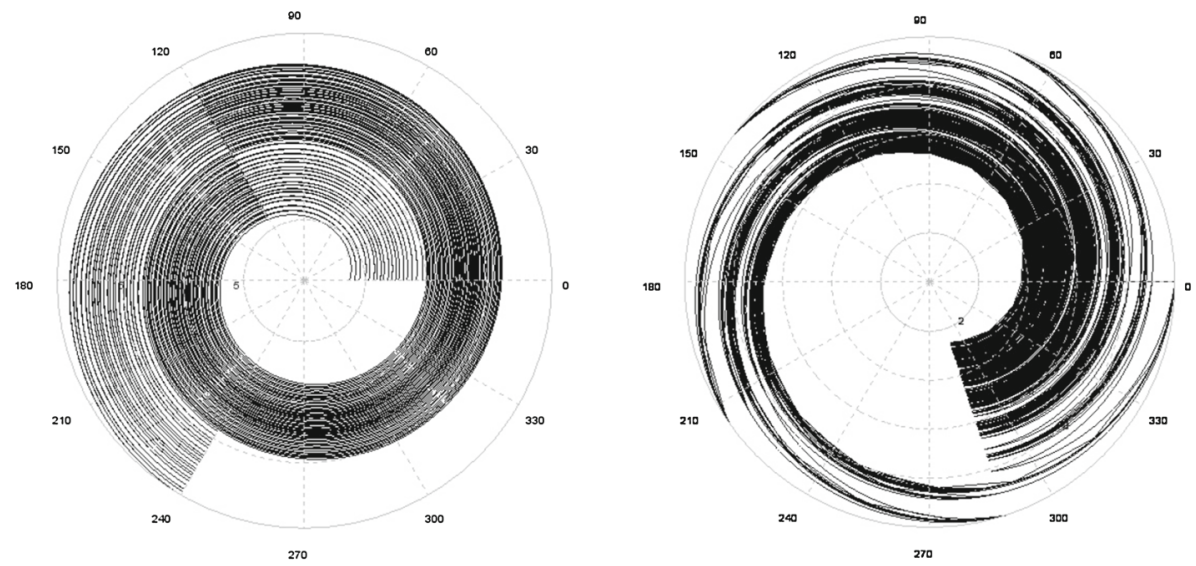

Fig. 1 Strange attractors, supporting annulus homeomorphisms with non-unique rotation numbers, obtained by a construction on an inverse limit of circles, as described in [17]

is topologically conjugate to the induced map on an inverse limit space based on a branched one-dimensional manifold, but Barge [5] proved that certain dynamical systems with Hénontype attractors cannot me modeled on inverse limits. In addition, authors' recent example of torus homeomorphism with an attracting pseudo-circle as Birkhoff-type attractor in [17] is non-differentiable and has infinite entropy (see Fig. 1).

\section{Preliminaries}

\subsection{Entropy}

Topological entropy is one of the most common measures of complexity of dynamical systems. The reader not familiar with this notion is referred to $[1,47]$ for more details. Below we recall only the main definitions related to this notion.

Given two finite open covers $\mathcal{U}, \mathcal{V}$ of $X$ we define the cover $\mathcal{U} \vee \mathcal{V}$ by

$$
\mathcal{U} \vee \mathcal{V}=\{U \cap V: U \in \mathcal{U}, V \in \mathcal{V}\}
$$

For each $n \geq 0$ we denote $T^{-n}(\mathcal{U})=\left\{T^{-n}(U): U \in \mathcal{U}\right\}$. Similarly, for any $0 \leq m \leq n$ define

$$
\mathcal{U}_{m}^{n}=T^{-m}(\mathcal{U}) \vee T^{-m-1}(\mathcal{U}) \vee \cdots \vee T^{-n}(\mathcal{U}) .
$$

Denote by $N(\mathcal{U})$ the minimal cardinality among all subcovers $\mathcal{V} \subseteq \mathcal{U}$ and then define

$$
h_{\text {top }}(f, \mathcal{U})=\limsup _{n \rightarrow+\infty} \frac{1}{n} \log N\left(\mathcal{U}_{0}^{n-1}\right) .
$$

Clearly, if $\mathcal{V}$ is a subcover of $\mathcal{U}$ then $h_{\text {top }}(f, \mathcal{U}) \leq h_{\text {top }}(f, \mathcal{V})$. The topological entropy of $(X, f)$ is defined by $h_{\text {top }}(f)=\sup _{\mathcal{U}} h_{\text {top }}(f, \mathcal{U})$ where the supremum is taken over all open covers $\mathcal{U}$ of $X$.

For any $n \in \mathbb{N}$, the $d_{n}$-distance between $x, y \in X$ is defined as

$$
d_{n}(x, y):=\max _{0 \leq i \leq n-1}\left\{d\left(f^{i} x, f^{i} y\right)\right\}
$$


Fix a set $A \subset X$ and $\varepsilon>0$. A set $S \subset A$ is $(n, \varepsilon)$-separated for $A$ if $d_{n}(x, y)>\varepsilon$ for any distinct $x, y \in S$. Denote $s_{n}(A, \varepsilon)=\sup \{|S|: S$ is $(n, \varepsilon)-$ separated for $A\}$ where $|S|$ denotes the cardinality of $S$.

For any compact set $A \subset X$ we define its topological entropy by

$$
h_{\text {top }}(f, A)=\lim _{\varepsilon \rightarrow 0} \lim _{n \rightarrow \infty} \frac{\log s_{n}(A, \varepsilon)}{n} .
$$

Since $X$ is compact, it is well known that $h_{\text {top }}(f)=h_{\text {top }}(f, X)$. If the map $f$ is clear from the context we simply write $h_{\text {top }}(A)$.

Entropy pairs were introduced by Blanchard in [12] and later studied by various authors. The main feature related to entropy pairs is that the dynamical system has positive topological entropy if and only if it has an entropy pair. An important generalization of this concept are entropy sets, introduced in [22]. A set $K \subset X$ with at least two points is an entropy set if for any finite open cover $\mathcal{U}$ of $X$ such that $K$ is not contained in the closure of any of its elements we have $h_{\text {top }}(f, \mathcal{U})>0$.

It is much easier to detect if a set is an entropy set, if we use the concept IE-tuples (the terminology comes from [35], but the concept appeared earlier in the literature, e.g. [29, Theorem 8.2]). We call a tuple $x=\left(x_{1}, \ldots, x_{k}\right) \in X^{k}$ an IE-tuple if for every product neighborhood $U_{1} \times \ldots \times U_{k}$ of $x$, the tuple $\left(U_{1}, \ldots, U_{k}\right)$ has an independence set $T \subset \mathbb{N}$ of positive upper density, that is for every finite subset $P \subset T$ and function $\zeta: P \rightarrow$ $\{1,2, \ldots, k\}$ we have $\bigcap_{j \in P} f^{-j}\left(U_{\zeta(j)}\right) \neq \emptyset$. Recall that the upper density of a set $A \subset \mathbb{N}$ is the number $\lim \sup _{n \rightarrow \infty} \frac{1}{n} \#(A \cap[0, n))$. It is known (see [13,29,35]) that a set $K$, which is not a singleton, is an entropy set iff every finite selection of its points $x_{1}, \ldots, x_{n} \in K$ forms an IE-tuple.

Note that the closure of an entropy set is an entropy set, so in this paper we will consider only closed entropy sets. By the same observation (and Zorn lemma), every entropy set is always contained in a maximal entropy set (in the sense of inclusion).

Positive entropy implies existence of entropy sets. It may happen however that there is no entropy set with entropy close to entropy of the map. The following simple example shows that entropy can be infinite, while every entropy set has finite entropy.

Example 2.1 Call a map $g$ on a nondegenerate interval $[a, b] n$-fold, $n \geq 2$ if

$$
g(a+j(b-a) / n)=a+(b-a)\left(1+(-1)^{j+1}\right) / 2
$$

for $j=0, \ldots, n$.

Put $I_{n}=\left[1 / 2^{n}, 1 / 2^{n-1}\right]$ for $n=1,2, \ldots$ and let $f:[0,1] \rightarrow[0,1]$ be defined by $f(0)=0$ and $\left.f\right|_{I_{n}}$ is $(2 n+1)$-fold map (see Fig. 2). Observe that $f$ is continuous and $h_{\text {top }}(f)=\infty$ since entropy of $n$-fold map is $\log n$.

But every entropy set of $f$ must be contained completely in one of the intervals $I_{n}$ and hence its entropy is finite.

\subsection{Graphs and Horseshoes}

A (topological) graph is a continuum $G$ which can be represented as a union of finitely many arcs, any two of them having at most one point in common. Each topological graph is homeomorphic to a simplical complex embedded in the Euclidean space $\mathbb{R}^{3}$ (see [40]), hence we may consider each graph $G$ endowed with the taxicab metric, that is, the distance between any two points of $G$ is equal to the length of the shortest arc in $G$ joining these points. A set $I \subseteq G$ is a closed interval if there is a homeomorphism $\varphi:[0,1] \rightarrow I$ such 
Fig. 2 Sketch of the graph of map $g$ in Example 2.1

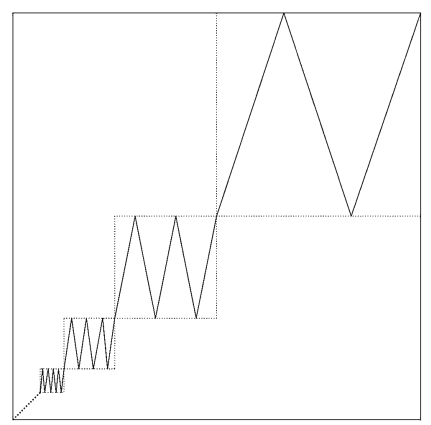

that the set $\varphi((0,1))$ is open in $G$. A path in a graph $G$ is any map $\omega:[0,1] \rightarrow G$. If $\omega:[0,1] \rightarrow G$ is a homeomorphism on its image and $q, r \in[0,1]$ then we say that $\omega([q, r])$ is an $\operatorname{arc}$ in $G$ and we denote it by $\langle a, b\rangle$, where $a=\omega(q)$ and $b=\omega(r)$. In such a case, without loss of generality, we shall always assume that $\omega$ is an isometry and we shall write $t_{1}<t_{2}$ (resp. $\left.t_{1} \leq t_{2}\right)$ for $t_{1}, t_{2} \in \omega([0,1])$ if $\omega^{-1}\left(t_{1}\right)<\omega^{-1}\left(t_{2}\right)$ (resp. $\left.\omega^{-1}\left(t_{1}\right) \leq \omega^{-1}\left(t_{2}\right)\right)$. We say that the set $(a, b)=\langle a, b\rangle \backslash\{a, b\}$ is an open arc. Note that sometimes $(a, b)$ is not open in $G$ hence it is not interior of $\langle a, b\rangle$ in $G$ (however it is an open set in $\langle a, b\rangle)$.

Given a map $f: G \rightarrow G$ and $\operatorname{arcs} I, J$ in $G$ we say that $I f$-covers $J$ if there exists an arc $K \subseteq I$ such that $f(K)=J$. Properties of $f$-covering relation presented below are adapted from [2, p. 590].

Lemma 2.2 Let $I, J, K, L \subseteq G$ be arcs, and let $f, g: G \rightarrow G$.

(1) If I $f$-covers $I$, then there exists $x \in I$ such that $f(x)=x$.

(2) If $I \subseteq K, L \subseteq J$ and $I f$-covers $J$, then $K f$-covers $L$.

(3) If I $f$-covers $J$ and $J g$-covers $K$, then $I(g \circ f)$-covers $K$.

(4) If $J \subseteq f(I)$, and $K_{1}, K_{2} \subseteq J$ are arcs such that $K_{1} \cap K_{2}$ is at most one point, then I $f$-covers $K_{1}$, or $I f$-covers $K_{2}$.

Fix an integer $s \geq 2$. An $s$-horseshoe for $f$ is an arc $I \subseteq G$ and subarcs $J_{1}, \ldots, J_{s}$ of $I$ with pairwise disjoint interiors, such that $f\left(S_{j}\right)=I$ for $j=1, \ldots, s$. An s-horseshoe is strong if in addition all the arcs $J_{i}$ are contained in the interior of $I$ and are pairwise disjoint. It is not hard to show that if $f$ has an $s$-horseshoe for $s \geq 4$ then it has an $(s-2)$-strong horseshoe [36].

Definition 2.3 Let $f: G \rightarrow G$ be a map of a graph $G$ with metric $d$ and let $\varepsilon>0$. A path $\omega:[0,1] \rightarrow G$ is $(f, \varepsilon)$-crooked, if there exist $s$ and $t$ with $0<s \leq t<1$ such that $d(f \circ \omega(s), f \circ \omega(1)) \leq \varepsilon$ and $d(f \circ \omega(t), f \circ \omega(0)) \leq \varepsilon$ (see Fig. 3).

Remark 2.4 It is an immediate consequence of the definition, that if for some $\varepsilon>0$ and $n>0$ the path $\omega:[0,1] \rightarrow G$ is $\left(f^{n}, \varepsilon\right)$-crooked then it is also $\left(f^{n+1}, \varepsilon\right)$-crooked.

\section{Graphs, Inverse Limits, Crookednes and Entropy Sets}

Given a map $f: X \rightarrow X$ on a metric space $X$, the inverse limit space $X_{f}=\lim _{\longleftarrow}(X, f)$ is the space given by

$$
X_{f}=\left\{\left(x_{1}, x_{2}, x_{3}, \ldots\right) \in X^{\mathbb{N}}: f\left(x_{i+1}\right)=x_{i}\right\} .
$$




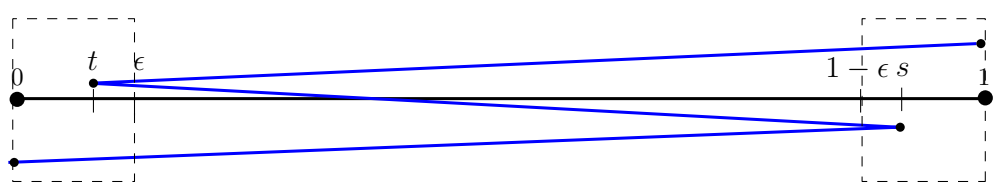

Fig. $3 \epsilon$-crooked arc

The topology of $X_{f}$ is induced from the product topology of $X^{\mathbb{N}}$, with the basic open sets in $X_{f}$ given by

$$
U_{\leftarrow}=\left(f^{i-1}(U), f^{i-2}(U), \ldots, U, f^{-1}(U), f^{-2}(U), \ldots\right),
$$

where $U$ is an open subset of the $i$-th factor space $X$. The map $f$ is called a bonding map and there is a natural homeomorphism $\sigma_{f}: X_{f} \rightarrow X_{f}$, called the shift homeomorphism, given by

$$
\sigma_{f}\left(x_{1}, x_{2}, x_{3}, \ldots\right)=\left(f\left(x_{1}\right), f\left(x_{2}\right), f\left(x_{3}\right), \ldots\right)=\left(f\left(x_{1}\right), x_{1}, x_{2}, \ldots\right) .
$$

It is well known that $\sigma_{f}$ preserves many dynamical properties of $f$. In particular, topological entropies of $f$ and $\sigma_{f}$ are the same (see [20] for details).

The following result of Brown [18, Lemma 3] will be crucial in the proof of our main results.

Lemma 3.1 Let $G$ be a topological graph. If the inverse limit $\lim (G, f)$ is hereditarily indecomposable then for every $\delta>0$ there is $n>0$ such that each path $\omega:[0,1] \rightarrow G$ is $\left(f^{n}, \delta\right)$-crooked.

We will also need an important extension of the result of Misiurewicz and Szlenk first proved for interval maps, connecting topological horseshoes with positive topological entropy [36, Theorem B].

Lemma 3.2 If $f: G \rightarrow G$ has positive topological entropy (i.e. $h_{\text {top }}(f)>0$ ) then there exist sequences $\left\{m_{i}\right\}_{i=1}^{\infty}$ and $\left\{k_{i}\right\}_{i=1}^{\infty}$ of positive integers such that for each $n$ the map $f^{m_{n}}$ has a $k_{n}$-horseshoe and

$$
\limsup _{n \rightarrow \infty} \frac{1}{m_{n}} \log \left(k_{n}\right)=h_{\text {top }}(f)
$$

If $\left\langle a^{\prime}, b^{\prime}\right\rangle$ is an arc, $a^{\prime}<a<b<b^{\prime}$ and $\varepsilon>0$ is such that $d\left(a, a^{\prime}\right) \geq \varepsilon$ and $d\left(b, b^{\prime}\right) \geq \varepsilon$ then, since distance on $G$ is given by arc length, there are points $a^{\prime} \leq a_{\varepsilon} \leq a$ and $b \leq b_{\varepsilon} \leq b^{\prime}$ such that $d\left(a, a_{\varepsilon}\right)=\varepsilon$ and $d\left(b, b_{\varepsilon}\right)=\varepsilon$. Therefore we will use the following natural notation $\langle a-\varepsilon, b+\varepsilon\rangle=\left\langle a_{\varepsilon}, b_{\varepsilon}\right\rangle$.

Lemma 3.3 Let $G$ be a topological graph, $\varepsilon>0$ be a number, and let $\langle a-\varepsilon, b+\varepsilon\rangle$ and $\langle c, d\rangle$ be arcs in $G$. Fix any even integer $k>0$ and let $\delta<\varepsilon / 6 k$. If $g: G \rightarrow G$ is a map such that $\langle a-\epsilon, b+\epsilon\rangle \subseteq g(\langle c, d\rangle)$ and each path in $G$ is $(g, \delta)$-crooked then there are points $p_{i}, l_{i} \in\langle c, d\rangle$ such that

$$
c \leq l_{1}<p_{1}<\ldots<l_{k}<p_{k} \leq d
$$

and such that one of the following conditions holds: 
(i) $g\left(l_{i}\right) \in\langle a-\varepsilon, a\rangle, g\left(p_{i}\right) \in\langle b, b+\varepsilon\rangle$ for all $1 \leq i \leq k$, or

(ii) $g\left(p_{i}\right) \in\langle a-\varepsilon, a\rangle, g\left(l_{i}\right) \in\langle b, b+\varepsilon\rangle$ for all $1 \leq i \leq k$.

Proof There are $c \leq l_{1}<p_{k} \leq d$ such that $g\left(l_{1}\right)=a-\varepsilon / 2, g\left(p_{k}\right)=b+\varepsilon / 2$ or $g\left(p_{k}\right)=a-\varepsilon / 2, g\left(l_{1}\right)=b+\varepsilon / 2$.

Let us assume that $g\left(l_{1}\right)=a-\varepsilon / 2, g\left(p_{k}\right)=b+\varepsilon / 2$ (for the second case the proof is the same, and corresponds to (ii)). Since any path in $G$ is $(g, \delta)$-crooked, there are $l_{1}<p_{1}<$ $l_{k}<p_{k}$ such that

$$
d\left(g\left(p_{1}\right), b+\varepsilon / 2\right)<\delta \text { and } d\left(g\left(l_{k}\right), a-\varepsilon / 2\right)<\delta .
$$

Next, let us assume that for some $j \geq 1, j<k / 2$ there are points

$$
c \leq l_{1}<p_{1}<\ldots<l_{j}<p_{j}<l_{k-j+1}<p_{k-j+1}<\ldots<l_{k}<p_{k} \leq d
$$

such that for $i=1,2, \ldots, j$ we have

$$
g\left(p_{k-i}\right), g\left(p_{i}\right) \in\langle b+\varepsilon / 2-2(i-1) \delta, b+\varepsilon / 2+2(i-1) \delta\rangle,
$$

and

$$
g\left(l_{k-i}\right), g\left(l_{i}\right) \in\langle a-\varepsilon / 2-2(i-1) \delta, a-\varepsilon / 2+2(i-1) \delta\rangle .
$$

Using again the fact that $g$ is $\delta$-crooked, there are

$$
p_{i}<l_{i+1}<p_{k-i}<l_{k-i+1}
$$

such that

$$
\begin{aligned}
g\left(l_{i+1}\right) & \in\left\langle g\left(l_{i}\right)-\delta, g\left(l_{i}\right)+\delta\right\rangle \\
& \subseteq\langle a-\varepsilon / 2-(2 i-1) \delta, a-\varepsilon / 2+(2 i-1) \delta\rangle,
\end{aligned}
$$

and

$$
\begin{aligned}
g\left(p_{k-i}\right) & \in\left\langle g\left(p_{k-i+1}\right)-\delta, g\left(l_{k-i+1}\right)+\delta\right\rangle \\
& \subseteq\langle b+\varepsilon / 2-(2 i-1) \delta, b+\varepsilon / 2+(2 i-1) \delta\rangle .
\end{aligned}
$$

Similarly, there are

$$
l_{i+1}<p_{i+1}<l_{k-i}<p_{k-i}
$$

such that

$$
\begin{aligned}
g\left(p_{i+1}\right) & \in\left\langle g\left(p_{k-i}\right)-\delta, g\left(l_{k-i}\right)+\delta\right\rangle \\
& \subseteq\langle b+\varepsilon / 2-2 i \delta, b+\varepsilon / 2+2 i \delta\rangle . \\
g\left(l_{k-i}\right) & \in\left\langle g\left(l_{i+1}\right)-\delta, g\left(l_{i+1}\right)+\delta\right\rangle \\
& \subseteq\langle a-\varepsilon / 2-2 i \delta, a-\varepsilon / 2+2 i \delta\rangle,
\end{aligned}
$$

By induction on $j=1,2, \ldots, k / 2$, we eventually obtain that there are $c \leq l_{1}<p_{1}<$ $\ldots<l_{k}<p_{k} \leq d$ such that for $i=1, \ldots, k$ we have

$$
g\left(l_{i}\right) \in\langle a-\varepsilon / 2-k \delta, a-\varepsilon / 2+k \delta\rangle \subseteq\langle a-\varepsilon, a\rangle
$$

and

$$
g\left(p_{i}\right) \in\langle b+\varepsilon / 2-k \delta, b+\varepsilon / 2+k \delta\rangle \subseteq\langle b, b+\varepsilon\rangle .
$$

The proof is complete. 
We have now collected enough tools to prove that the entropy of maps with hereditarily indecomposable inverse limits cannot be bounded, because the size of horseshoes grows very fast when the iteration increases.

Lemma 3.4 Let $G$ be a topological graph, assume that the inverse limit $\lim (G, f)$ is hereditarily indecomposable. Let $J, L$ be closed intervals such that $J \subset$ int $L \overleftarrow{\text { and }}$ there is $n>0$ such that $J f^{n}$-covers $L$. Then for every $\alpha>0$ there are $m, k>0$ and pairwise disjoint intervals $J_{0}, J_{1}, \ldots, J_{k} \subset J$ such that $J_{i} f^{m n}$-covers $L$ for each $i=0, \ldots, k$ and

$$
\frac{1}{m n} \log (k)>\alpha \text {. }
$$

Proof Fix any integer $\tilde{k}>0$ such that $\frac{1}{n} \log (\tilde{k})>\alpha$. Denote $g=f^{n}$ and $r=\tilde{k}^{2}$. Without loss of generality we may assume that $g(J)=L$. Therefore, there are $\varepsilon>0$ and $a<b$ such that $J \subseteq(a, b) \subset(a-2 \varepsilon, b+2 \varepsilon) \subset L$. Fix any $\delta<\varepsilon / 6 r$ and let $s$ be provided by Lemma 3.1, that is each path in $G$ is $\left(g^{s}, \delta\right)$-crooked. Arc $J$ for $g$ can be "iterated" for $g^{s}$, that is we may replace $J$ by its subinterval $J^{\prime}$ in such a way that $g^{s}\left(J^{\prime}\right)=L$.

Let us apply Lemma 3.3 to $\left\langle c^{\prime}, d^{\prime}\right\rangle=J^{\prime} \subset J=\langle c, d\rangle$ obtaining numbers $c^{\prime} \leq l_{1}<$ $p_{1}<\ldots<l_{r}<p_{r} \leq d^{\prime}$ and for simplicity of notation suppose that $g^{s}\left(l_{j}\right) \in\langle a-\varepsilon, a\rangle$ and $g^{s}\left(p_{j}\right) \in\langle b, b+\varepsilon\rangle$ for all $j$ (the arguments for the symmetric case $g^{s}\left(p_{j}\right) \in\langle a-\varepsilon, a\rangle$ and $g^{s}\left(l_{j}\right) \in\langle b, b+\varepsilon\rangle$ are the same). Then, since we may view $g^{s}: J^{\prime} \rightarrow L$ as a map between subintervals of the real line, a simple argument shows that each arc $\left\langle l_{j}, p_{j}\right\rangle g^{s}$-covers $J$. Hence, there are $\operatorname{arcs} J_{1}, \ldots, J_{r}$ such that $g^{s}\left(J_{i}\right)=J$ and since $g(J)=L$ we obtain that each $J_{i} g^{s+1}$-covers $L$. But each path in $G$ is also $\left(g^{s+1}, \delta\right)$-crooked, so we can continue this procedure recursively. After $s$ steps, we obtain pairwise disjoint $\operatorname{arcs} J_{i_{1}, i_{1}, i_{2}, \ldots, i_{s}} \subseteq J$, $i_{1}, \ldots, i_{s} \in\{1, \ldots, r\}$, such that each arc $J_{i_{1}, i_{2}, \ldots, i_{s}} g^{2 s}$-cover $L$. Then there are at least $r^{s}$ pairwise disjoint intervals which $g^{2 s}$-cover $\langle a-\varepsilon, b+\varepsilon\rangle$.

But then easy calculation yields that

$$
\frac{1}{n} \frac{1}{2 s} \log \left(r^{s}\right) \geq \frac{1}{2 n} \log (r) \geq \frac{1}{n} \log (\tilde{k})>\alpha
$$

hence it is enough to put $k=r^{s}$ and $m=2 s$ to end the proof.

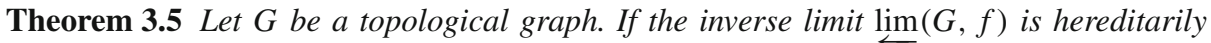

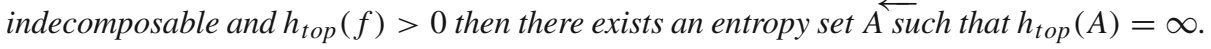

Proof Take any $\alpha>0$ such that $h_{\text {top }}(f)>\alpha$. By Lemma 3.2 there are $s, k>0$ such that $\frac{1}{s} \log (k)>\alpha$ and a strong $(k+1)$-horseshoe $J_{0}, \ldots, J_{k} \subset$ int $L$ for $f^{s}$.

Next we will present a method for enlarging invariant sets induced by horseshoes. For this purpose, suppose that we have constructed pairwise disjoint intervals $J_{0}, \ldots, J_{k} \subset$ int $L$ and there is $s>0$ such that each $J_{i} f^{s}$-covers $L$. By Lemma 3.4 for every $N>0$ there are $r, m>0$ and pairwise disjoint intervals $I_{0}, I_{1}, \ldots, I_{r} \subset J_{0}$ such that $I_{i} f^{s m}$-covers $L$ for each $i=0, \ldots, r$, and

$$
\frac{1}{s m} \log (r)>N \alpha .
$$

Furthermore, by the definition of horseshoe, for every $w \in\{1, \ldots, k\}^{m}$ there is an interval $J(w)$ such that $f^{s j}(J(w)) \subset J_{w(j)}$ for all $0 \leq j<m$ and $f^{s m}(J(w))=L$. Simply we put $J(w)=\bigcap_{j=0}^{m-1} f^{-s j}\left(J_{w_{j}}\right)$. In particular intervals $J(w), w \in\{1, \ldots, k\}^{m}$ form a (strong) horseshoe for $f^{s m}$ in $L$. Intervals $I_{0}, I_{1}, \ldots, I_{r} \subset J_{0}$, hence we can add them to the collection of intervals $J(w)$ obtaining a larger horseshoe (with $k^{m}+r+1$ elements). 
Now we will present a construction from the proof of Proposition II.15 in [15]. While the construction is standard, we will need to make some essential modifications in two places, so we need to recall this construction briefly. For each $a \in\{1, \ldots, k\}$ write $J(a)=J_{a}$. Since $J_{1}, \ldots, J_{k}$ is a strong horseshoe for $f^{s}$, for every $a, b \in\{1, \ldots, k\}$ we can find an interval $J(a b) \subset J(a)$ such that $f(J(a b))=J(b)$.

Next, fix $n$ and assume that for any sequence $a_{1}, \ldots, a_{n} \in\{1, \ldots, k\}$ we have already constructed intervals

$$
J\left(a_{1} a_{2} \ldots a_{n}\right) \subset J\left(a_{1} a_{2} \ldots a_{n-1}\right) \subset \ldots \subset J\left(a_{1} a_{2}\right) \subset J\left(a_{1}\right)
$$

and $f^{s}\left(J\left(a_{1} a_{2} \ldots a_{k}\right)\right)=J\left(a_{2} \ldots a_{k}\right)$ for every $2 \leq k \leq n$

By the above construction, for any sequence $\xi \in\{1, \ldots, k\}^{\mathbb{N}}$ the set $A_{\xi}$ $=\bigcap_{i=1}^{\infty} J\left(\xi_{1} \xi_{2} \ldots \xi_{i}\right)$ is a nonempty interval, because it is an intersection of a nested sequence of closed intervals. Let $\Lambda$ be a set that consists of endpoints of all possible sets $A_{\xi}$. That way we obtain an invariant set $\Lambda \subset L$ for $f^{s}$ and a continuous onto map $\pi: \Lambda \rightarrow \Sigma_{k}$ such that $\pi$ is one-to-one for all but countably many points, $\sigma: \Sigma_{k} \rightarrow \Sigma_{k}$ is the full shift on $k$ symbols and $\pi \circ f^{s}=\sigma \circ \pi$. Namely, $\pi$ sends endpoints of $A_{\xi}$ to $\xi$ but it may happen that some (but at most countably many) intervals $A_{\xi}$ are nondegenerate.

We have to make one important remark at this point. In the case that $A_{\xi}$ is an interval, it could happen that one of its endpoints is isolated in $\Lambda$. To remedy this situation we can take $x \in \Lambda$ such that $\pi(x)$ has dense orbit in $\Sigma_{k}$ and $\pi^{-1} \pi(x)$ is a singleton. Then $x$ is recurrent, $\hat{\Lambda}=\overline{\left\{f^{s n}(x): n \geq 0\right\}}$ is a closed $f^{s}$-invariant set without isolated points, and $\pi(\hat{\Lambda})=\Sigma_{k}$. Therefore in what follows, we assume that sets $\Lambda$ constructed by the use of horseshoe are without isolated point.

Furthermore observe that if we take intervals $J_{w}=J(w), w \in\{1, \ldots, k\}^{m}$ then we obtain a strong horseshoe for $f^{s m}$. If we fix $w_{1}, w_{2} \in\{1, \ldots, k\}^{m}$ then by the construction $J\left(w_{1} w_{2}\right) \subset J_{w_{1}}$ and $f^{s m}\left(J\left(w_{1} w_{2}\right)\right)=J_{w_{2}}$. Hence, in the construction of invariant set for $f^{s m}$ and horseshoe $J_{w}, w \in\{1, \ldots, k\}^{m}$ we can take $J\left(\left(w_{1}\right)\left(w_{2}\right)\right)=J\left(w_{1} w_{2}\right)$ and extend it inductively on the longer sequences of symbols $J\left(\left(w_{1}\right)\left(w_{2}\right) \ldots\left(w_{n}\right)\right)=J\left(w_{1} w_{2} \ldots w_{n}\right)$. Condition (1) ensures that

$$
\begin{aligned}
A_{\xi} & =\bigcap_{i=1}^{\infty} J\left(\xi_{1} \xi_{2} \ldots \xi_{i}\right)=\bigcap_{i=1}^{\infty} J\left(\xi_{1} \xi_{2} \ldots \xi_{n i}\right) \\
& =\bigcap_{i=1}^{\infty} J\left(\left(\xi_{1} \ldots \xi_{n}\right)\left(\xi_{n+1} \ldots \xi_{2 n}\right) \ldots\left(\xi_{n(i-1)+1} \ldots \xi_{n i}\right)\right)
\end{aligned}
$$

using horseshoe $J(w), w \in\{1, \ldots, k\}^{m}$ we obtain the same set $\Lambda$, but now it is invariant for $f^{s m}$ and connecting factor map is now $\hat{\pi}: \Lambda \rightarrow \Sigma_{k^{m}}$. This immediately implies, that using the above extension of horseshoe, if $\Lambda$ was $f^{s}$-invariant set produced using horseshoe $J_{1}, \ldots, J_{k}$ and $\Gamma$ is $f^{s m}$-invariant set constructed using horseshoe consisting of sets $J(w), w \in\{1, \ldots, k\}^{m}$ together with $I_{i}, 0 \leq i \leq r$ then $\Lambda \subset \Gamma$ and we can extend $\hat{\pi}$ to a map $\hat{\pi}: \Gamma \rightarrow \Sigma_{k^{m}+r}$. As before, we may assume that $\Gamma$ does not have isolated points.

By the construction $h_{\text {top }}(\Lambda) \geq \frac{1}{s} \log (k)$ and

$$
h_{\text {top }}(\Gamma)=h_{\text {top }}\left(\left.f^{s m}\right|_{\Gamma}\right)=\frac{1}{s m} \log \left(k^{m}+r\right)>\frac{1}{s m} \log (r) .
$$

Recall that if $\pi$ is a factor map, and $x_{1}, \ldots, x_{n}$ is an IE-tuple, then the fiber $\pi \times \ldots \times$ $\pi\left(\left\{\left(x_{1}, \ldots, x_{n}\right)\right\}\right)$ contains at least one IE- $n$-tuple (see elementary properties of entropy 
tuples, e.g. in [29]). But $\pi$ is one-to-one on all but countably many points and sets $\Lambda, \Gamma$ do not have isolated points, hence it is not hard to see that $\Lambda$ (the same for $\Gamma$ ) is an entropy set, since $\Sigma_{k}$ is an entropy set. Namely, in arbitrarily neighborhood of any tuple of distinct points $x_{1}, \ldots, x_{n}$ in $\Lambda$ we can find a tuple which is IE $n$-tuple, and hence $\left(x_{1}, \ldots, x_{n}\right)$ is IE $n$-tuple itself. Then by the above procedure we can construct a sequence of closed entropy sets $\Lambda_{1} \subset \Lambda_{2} \subset \ldots$ such that $h_{\text {top }}\left(\Lambda_{n}\right) \geq n \alpha$. Now, if we denote $D=\overline{\bigcup_{j} \Lambda_{j}}$ then clearly $D$ is also an entropy set, but now $h_{\text {top }}(D)=\infty$.

As a consequence of Lemma 3.4 we easily obtain the following result. It is a direct consequence of Theorem 3.5, but proving it this way would be overcomplicated.

Corollary 3.6 Let $G$ be a topological graph. If the inverse limit $\underset{\longleftarrow}{\lim }(G, f)$ is hereditarily indecomposable and $h_{\text {top }}(f)>0$ then $h_{\text {top }}(f)=\infty$.

Proof If $h_{\text {top }}(f)>0$ then by Lemma 3.2 there is $n$ such that $f^{n}$ has a 3-horseshoe. In particular, if we denote by $J$ the middle interval of three intervals defining 3-horseshoe, then $J$ satisfies assumptions of Lemma 3.4 and hence for every $\alpha>0$ there are $m, k$ such that

$$
h_{\text {top }}(f)=\frac{1}{m n} h_{\text {top }}\left(f^{m n}\right) \geq \frac{1}{m n} \log (k)>\alpha .
$$

The proof is finished.

Corollary 3.6 has an important consequence for smooth dynamical systems on compact finite-dimensional Riemannian manifolds, when combined with the following result of Ito.

Theorem 3.7 (Ito [31]) Let $(M, g)$ be a compact $n$-dimensional Riemannian manifold and $F: M \rightarrow M a C^{1}$-diffeomorphism. Then $h_{\text {top }}(F)$, the topological entropy of $F$, is finite.

Corollary 3.8 Let $(M, g)$ be a compact $n$-dimensional Riemannian manifold and $F: M \rightarrow$ $M$ be a homeomorphism with an invariant hereditarily indecomposable continuum $X$; i.e. $F(X)=X$. If $F \mid X$ is conjugate to a shift homeomorphism on a graph inverse limit $\lim _{\longleftarrow}(G, f)$ then either $h_{\text {top }}(F)=0$ or $F$ is non-differentiable.

\section{Circle-Like Hereditarily Indecomposable Continua and Entropy}

There is a well known example of Henderson [27], who proved that shift homeomorphism on the pseudo-arc can have zero topological entropy. For the pseudo-circle, there is also an important example of Handel [26] who constructed a homeomorphism on the pseudo-circle (extendable to the whole plane) with zero topological entropy. Related results in complex dynamics were obtained by Herman [28] and Chéritat [21]. The natural question is whether the example of [27] can be somehow generalized to obtain the pseudo-circle as an inverse limit with one bonding map and with zero entropy. So far such an example was not constructed. We will show, that the reason for lack of such an example is very natural. Simply, such an example does not exist. This situation is in some sense similar to the case of Hénon-type attractors. First, Williams showed that every hyperbolic, one-dimensional, expanding attractor for a discrete dynamical system is topologically conjugate to the induced map on an inverse limit space based on a branched one-manifold [48]. Later Barge showed, however, that certain dynamical systems with Hénon-type attractors cannot me modeled on inverse limits [5].

In this section we are going to show that if $\Lambda_{f}=\lim _{\leftarrow}\left(\mathbb{S}^{1}, f\right)$ is hereditarily indecomposable, but $\Lambda_{f}$ is not the pseudo-arc, then $h_{\text {top }}(f)=\overleftarrow{\infty}$. In particular, one cannot construct 
a finite entropy homeomorphism on the pseudo-circle, or pseudo-solenoids conjugate to the shift homeomorphism on $\lim \left(\mathbb{S}^{1}, f\right)$. Recall that hereditarily indecomposable circle-like continua were classified by Fearnley [25] and Rogers [45]. The classification depends on the degree of the bonding map. According to the classification any continuum in this class is one of the following:

- $(\operatorname{deg}(f)=0)$ Knaster's pseudo-arc: the unique arc-like hereditarily indecomposable continuum.

- $(|\operatorname{deg}(f)|=1)$ R.H. Bing's pseudo-circle: the unique plane separating hereditarily indecomposable circle-like continuum.

- $(|\operatorname{deg}(f)|>1)$ Nonplanar pseudosolenoids: an uncountable family of continua.

Note that every proper subcontinuum of either hereditarily indecomposable circle-like continuum must be arc-like and hereditarily indecomposable so it is homeomorphic to the pseudo-arc.

In what follows we will use some standard fact on rotation numbers and sets for maps on the circle. The reader not familiar with this topic is refereed to Chapter 3 in [1].

We will need the following result of Auslander and Katznelson [3], which extends the classification of periodic-point-free circle homeomorphisms, and implies that every periodicpoint-free circle map is semi-conjugate to an irrational rotation.

Theorem 4.1 Suppose $f: \mathbb{S}^{1} \rightarrow \mathbb{S}^{1}$ is such that $\operatorname{Per}(f)=\emptyset$. Then

(1) for every $x \in \mathbb{S}^{1}$ there is an interval $J_{x}=\left[a_{x}, b_{x}\right]$ such that $x \in J_{x}$,

(2) $f\left(J_{x}\right)$ is an interval with endpoints $f\left(a_{x}\right)$ and $f\left(b_{x}\right)$,

(3) $f^{m}(x) \notin f\left(J_{x}\right)$ for $m>1$,

(4) $f^{m}\left(J_{x}\right) \cap J_{f^{m}(x)}$ for $m=1,2,3, \ldots$,

(5) intervals $J_{f^{m}(x)}$ for $m=0,1,2, \ldots$ are pairwise disjoint,

(6) if $f(x)=f(y)$ then $J_{x}=J_{y}$,

(7) the sets $\mathcal{J}=\left\{J_{x}: x \in \mathbb{S}^{1}\right\}$ form a decomposition of $\mathbb{S}^{1}$; i.e. $J_{x}=J_{y}$ or $J_{x} \cap J_{y}=\emptyset$ for all $x, y \in \mathbb{S}^{1}$,

(8) for at most countably many $x$ we have $J_{x} \neq\{x\}$,

(9) $J_{x}=J_{y}$ if and only if $J_{f^{n}(x)}=J_{f^{n}(y)}$ for some (every) $m=0,1,2, \ldots$.

Theorem 4.2 Suppose $f: \mathbb{S}^{1} \rightarrow \mathbb{S}^{1}$ and $\operatorname{Per}(f)=\emptyset$. Then $\Lambda_{f}=\lim ^{\lim }\left(\mathbb{S}^{1}, f\right)$ is decomposable.

Proof Let $f$ be as above and let $\mathcal{J}$ be the collection of intervals guaranteed by (7) in Theorem 4.1. Without loss of generality we may assume that there is an $x \in \mathbb{S}^{1}$ such that $J_{x} \neq\{x\}$, since otherwise by (6) in Theorem $4.1 f$ would be a homeomorphism and $\Lambda_{f}=\mathbb{S}^{1}$. Additionally observe that $f^{-n}(z) \neq \emptyset$ for every $z \in \mathbb{S}^{1}$ as otherwise it is not hard to see that $f$ has a fixed point.

First we claim that $f^{-n}\left(J_{x}\right)$ is an interval in $\mathcal{J}$ for every $n$. To see this, fix any $n>0$ and any $y, z \in f^{-n}\left(J_{x}\right)$. By Theorem 4.1 (4) we have $f^{n}\left(J_{y}\right) \cap J_{x} \neq \emptyset$ and $f^{n}\left(J_{z}\right) \cap J_{x} \neq \varnothing$ which by Theorem 4.1(7) gives $f^{n}\left(J_{y}\right)=f^{n}\left(J_{z}\right)=J_{x}$. The proof of the claim is completed by Theorem 4.1 (9).

Put $K_{n}=f^{-n}\left(J_{x}\right)$. Since $K_{n}$ is an element of $\mathcal{J}$, without loss of generality we can write $K_{n}=\left[a_{n}, b_{n}\right]$ and $f\left(a_{n}\right)=a_{n-1}$ and $f\left(b_{n}\right)=b_{n-1}$. Put $L_{n}=\left\{a_{n}, b_{n}\right\} \cup\left(\mathbb{S}^{1} \backslash K_{n}\right)$. Then $L_{n}$ is an interval,

$$
f\left(L_{n}\right)=\left\{f\left(a_{n}\right), f\left(b_{n}\right)\right\} \cup\left(\mathbb{S}^{1} \backslash f\left(K_{n}\right)\right)=\left\{a_{n-1}, b_{n-1}\right\} \cup\left(\mathbb{S}^{1} \backslash K_{n-1}\right)=L_{n-1}
$$


and hence the following two maps are onto: $\left.f\right|_{K_{n}}: K_{n} \rightarrow K_{n-1},\left.f\right|_{L_{n}}: L_{n} \rightarrow L_{n-1}$ for $n=1,2, \ldots$. Therefore the following inverse limits

$$
\begin{aligned}
& \Gamma=\lim ^{\longleftarrow}\left(K_{n},\left.f\right|_{K_{n}}: n \geq 0\right), \\
& \Lambda=\lim _{(}\left(L_{n},\left.f\right|_{L_{n}}: n \geq 0\right)
\end{aligned}
$$

are arc-like continua such that $\Lambda_{f}=\Gamma \cup \Lambda$, in particular $\Lambda_{f}$ is decomposable.

Although this is not essential for the reasoning in the present paper, note that periodicpoint-free circle maps are of degree 1 (see [23]).

Theorem 4.3 If $\Lambda_{f}=\lim _{(}\left(\mathbb{S}^{1}, f\right)$ is hereditarily indecomposable and $\Lambda_{f}$ is not the pseudoarc (i.e. $\operatorname{deg}(f) \neq 0$ ) then $h_{\text {top }}(f)=\infty$.

Proof Suppose $\Lambda_{f}=\lim \left(\mathbb{S}^{1}, f\right)$ is hereditarily indecomposable and $|\operatorname{deg}(f)|>0$. Then $\Lambda_{f}$ is either the pseudo-circle or it is nonplanar. If $\Lambda_{f}$ is nonplanar then $\operatorname{deg}(f)=n$, for some $|n|>1$ (see [45, Theorem 9]). Consequently $h_{\text {top }}(f) \geq \log |n|$ by [1] and further, by Theorem 3.6, we obtain that $h_{\text {top }}(f)=\infty$.

Now suppose $\operatorname{deg}(f)= \pm 1$. By Theorem 4.2 we have $\operatorname{Per}(f) \neq \varnothing$. By [30, Theorem 15, p.9] for any integer $m>0$ we have

$$
\lim _{\longleftarrow}\left(\mathbb{S}^{1}, f\right)=\lim _{\longleftarrow}\left(\mathbb{S}^{1}, f^{m}\right)
$$

and $h_{\text {top }}\left(f^{m}\right)=m \cdot h_{\text {top }}(f)$, hence without loss of generality we may assume that $\operatorname{deg}(f)=1$ and Fix $(f) \neq \emptyset$. Clearly, for any $m$ we have $h_{\text {top }}(f)=\infty$ if and only if $h_{\text {top }}\left(f^{m}\right)=\infty$.

Let $\hat{f}: \mathbb{R} \rightarrow \mathbb{R}$ be a lift of $f$ to the universal covering $(\mathbb{R}, \tau)$, where $\tau(t)=(\cos (t), \sin (t))$ and $\mathbb{S}^{1}$ is the unit circle in the plane, centered at the origin. It is known that $\hat{f}$ can be extended to $\hat{\mathbb{R}}=\mathbb{R} \cup\{+\infty,-\infty\}$, with $\hat{f}( \pm \infty)= \pm \infty$. Because $\hat{\mathbb{R}}$ is homeomorphic to $[0,1]$ and $\lim _{(}(\mathbb{R}, \hat{f})$ is a universal covering of $\Lambda_{f}$ with the covering map given by $\hat{\tau}\left(x_{1}, x_{2}, x_{3}, \ldots\right)$ $=\left(\tau\left(x_{1}\right), \tau\left(x_{2}\right), \tau\left(x_{3}\right), \ldots\right)$ (see [17, Lemma 3.8]), we get that $\lim _{\leftarrow}(\hat{\mathbb{R}}, \hat{f})$ is the pseudo-arc [10]. Since $\operatorname{Fix}(f) \neq \emptyset, \hat{f}$ can be chosen in such a way so that $\overleftarrow{\operatorname{Fix}}(\hat{f}) \neq \emptyset$. Indeed, any lift of $f$ is uniquely determined by specifying where it sends a single point, and if $c$ is a fixed point of $f$ then there is $\hat{c} \in[0,2 \pi]$ such that $\tau(\hat{c})=c$, hence it is enough to set $\hat{c}=\hat{f}(\hat{c})$.

Set $K_{i}=\hat{f}^{i}([0,2 \pi])$ and note that $\hat{c} \in K_{i}$ for every $i$. We shall show that the rotation set $\rho(f)$ is nondegenerate. To demonstrate this, we claim that

$$
\lim _{i \rightarrow \infty} \sup \operatorname{diam}\left(K_{i}\right)=\infty \text {. }
$$

In order to prove (2), first we claim that

$$
\lim _{i \rightarrow \infty} \sup \operatorname{diam}\left(K_{i}\right) \neq 0 \text {. }
$$

Recall that $f$ is an onto map, and if $B$ is an interval of length less than $2 \pi$ then $\tau(B)$ is a proper subset of $\mathbb{S}^{1}$. Then it is enough to observe that

$$
\tau\left(K_{1}\right)=\tau(\hat{f}([0,2 \pi]))=f(\tau([0,2 \pi]))=f\left(\mathbb{S}^{1}\right)=\mathbb{S}^{1},
$$

so we must have $\operatorname{diam}\left(K_{1}\right) \geq 2 \pi$. Then working by induction, using the relation $K_{i+1}$ $=\hat{f}\left(K_{i}\right)$, we obtain that $\operatorname{diam}\left(K_{i}\right) \geq 2 \pi$ for every $i>0$.

Next, let us suppose that $\lim _{i \rightarrow \infty} \sup \operatorname{diam}\left(K_{i}\right)<\infty$. Set $K=\bigcup_{i=1}^{\infty} K_{i}$ and note that $K$ is connected, bounded and $\hat{c} \in K_{i}$ for every $i$. Now define sets $L_{i}$ for $i=1,2, \ldots$ as follows. Denote by $L_{1}$ a component of $\hat{f}^{-1}(K)$ such that $\hat{c} \in L_{1}$, and then for $i>1$ take as the set $L_{i}$ a component of $\hat{f}^{-1}\left(L_{i-1}\right)$ that contains $\hat{c}$. Finally put $L=\operatorname{cl}\left(\bigcup_{i \geq 0} L_{i}\right)$. Note that $K \subseteq L$. 
It is easy to see that $L$ is connected, as $\hat{c} \in L_{i}$ for every $i$. We claim that $L$ is bounded. Indeed, since $\hat{f}(x+2 k \pi)=\hat{f}(x)+2 k \pi$ we must have $\sup _{x \in[0,2 \pi]} \operatorname{diam}\left(\left\{\hat{f}^{n}(x): n \geq 0\right\}\right)$ $=\sup _{x \in \mathbb{R}} \operatorname{diam}\left(\left\{\hat{f}^{n}(x): n \geq 0\right\}\right)$. Since $K$ is bounded we must have $\sup _{x \in[0,2 \pi]} \operatorname{diam}\left(\left\{\hat{f}^{n}(x)\right.\right.$ : $n \geq 0\})<M$ for some real number $M$. But now, if $p, q \in L_{i}$ then

$$
\operatorname{diam}(K) \geq d\left(f^{i}(p), f^{i}(q)\right) \geq d(p, q)-2 M
$$

and hence $\operatorname{diam}\left(L_{i}\right) \leq \operatorname{diam}(K)+2 M$, which gives $\operatorname{diam}(L) \leq 2 \operatorname{diam}(K)+4 M$. By the definition $\hat{f}\left(L_{i}\right) \subset L_{i-1}$ for $i>1$ and for every $n$ we have

$$
\bigcup_{i=n+1}^{\infty} K_{i} \subset \hat{f}^{n}(K) \subset \hat{f}^{n}(L) \subset \hat{f}^{n-1}(L) \subset \ldots \subset L .
$$

If we denote $K_{i}=\left[p_{i}, q_{i}\right]$ and let $p=\liminf _{i \rightarrow \infty} p_{i}, q=\lim \sup _{i \rightarrow \infty} q_{i}$. Then

$$
\hat{c} \in[p, q] \subset \bigcup_{i=n+1}^{\infty} K_{i}
$$

for every $n>0$ and $q-p \geq 2 \pi$.

Then if we denote $S=\bigcap_{i=0}^{\infty} \hat{f}(L)$ then $S$ is a continuum such that $\hat{f}(S)=S$ and $[p, q] \subset S$, so $S$ is a closed nondegenerate interval in $\mathbb{R}$. Now let $\Lambda_{S}=\lim _{\longleftarrow}\left(S,\left.\hat{f}\right|_{S}\right)$. Since $\Lambda_{S} \subseteq \lim (\mathbb{R}, \hat{f})$, the continuum $\Lambda_{S}$ is the psuedo-arc. But

$$
\mathbb{S}^{1}=\tau([0,2 \pi]) \subseteq \tau([p, q]) \subseteq \tau(S)
$$

and so $\hat{\tau}\left(x_{1}, x_{2}, x_{3}, \ldots\right)=\left(\tau\left(x_{1}\right), \tau\left(x_{2}\right), \tau\left(x_{3}\right), \ldots\right)$ defines a continuous onto map from the pseudo-arc $\Lambda_{S}$ onto the pseudo-circle $\Lambda_{f}$. But the pseudo-circle cannot be obtained as the image of the pseudo-arc via a continuous map [46], which is a contradiction. This proves that $\lim _{i \rightarrow \infty} \sup \operatorname{diam}\left(K_{i}\right)=\infty$, completing the proof of (2).

Because $\hat{f}$ has a fixed point in $[0,2 \pi]$ we get by (2) that the rotation set $\rho(\hat{f})$ is nondegenerate. This implies in turn that $h_{\text {top }}(f)>0$ (see [39]) and so, again by Theorem 3.6, we obtain that $h_{\text {top }}(f)=\infty$ which ends the proof.

The following theorem follows from the above proof.

Theorem 4.4 Suppose $f: \mathbb{S}^{1} \rightarrow \mathbb{S}^{1}$ is a map with $\operatorname{deg}(f)=1$. If $\Lambda_{f}=\lim _{(}\left(\mathbb{S}^{1}, f\right)$ is hereditarily indecomposable then the rotation set $\rho(f)$ is nondegenerate.

In [17] the authors showed that there exists a 2-torus homeomorphism $h$ homotopic to the identity with an attracting R.H. Bing's pseudocircle $C$ such that the rotation set of $h \mid C$ is not a unique vector. It follows from Theorem 4.3 that the topological entropy of this homeomorphism is infinite.

In [46] Rogers considered a special class of circle-like continua which he called selfentwined. Among the many results, he showed that the pseudo-circle is self-entwined, any continuum in this class is indecomposable and no self-entwined continuum is a continuous image of the pseudo-arc. Adapting the proof of Theorem 4.3 and properties of self-entwined continua sketched above, we get the following.

Theorem 4.5 Suppose $\Lambda_{f}=\lim _{(}\left(\mathbb{S}^{1}, f\right)$ is self-entwined. Then $h(f)>0$.

Proof Since $\Lambda_{f}$ is indecomposable, by Theorem 4.2, $f$ must have a periodic point. Again, without loss of generality assuming that $f$ has a fixed point we can consider $\hat{f}: \mathbb{R} \rightarrow \mathbb{R}$, a lift 
of $f$ to the universal covering $(\mathbb{R}, \tau)$. Then $\hat{f}$ can be extended to $\hat{\mathbb{R}}=\mathbb{R} \cup\{+\infty,-\infty\}$, with $\hat{f}( \pm \infty)= \pm \infty$. Because $\hat{\mathbb{R}}$ is homeomorphic to $[0,1]$ and $\lim (\mathbb{R}, \hat{f})$ is a universal covering of $\Lambda_{f}$ with the covering map given by $\hat{\tau}\left(x_{1}, x_{2}, x_{3}, \ldots\right) \stackrel{\leftarrow}{=}\left(\tau\left(x_{1}\right), \tau\left(x_{2}\right), \tau\left(x_{3}\right), \ldots\right)$ (see [17, Lemma 3.8]), we get that $A=\lim (\hat{\mathbb{R}}, \hat{f})$ is an arc-like continuum. Now if we assume by

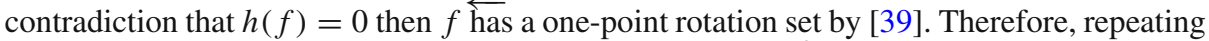
arguments from the proof of Theorem 4.3, there is a closed $\hat{f}$-invariant arc $S \subset \mathbb{R}$ such that arc-like continuum $\Lambda_{S}=\lim _{(}\left(S,\left.\hat{f}\right|_{S}\right) \subset A$ satisfies $\hat{\tau}\left(\Lambda_{S}\right)=\Lambda_{f}$. In this case $\Lambda_{f}$ would be a continuous image of an arc-like continuum. But since every arc-like continuum is a continuous image of the pseudo-arc [24] this leads to a contradiction with Roger's result that no self-entwined continuum is such an image and the proof is complete.

Acknowledgements The authors are grateful to the anonymous referee for his careful reading of this paper and many valuable suggestions. The authors express many thanks to Henk Bruin for fruitful discussions during Workshops on Complexity and Dimension theory of Skew Products Systems held at the Erwin Schrödinger International Institute for Mathematical Physics (ESI) in Vienna, in September of 2013, as well as Henk Bruin's visit at the AGH University of Science and Technology in Kraków, in November of 2013. The authors are grateful for the kind hospitality of the institute during their stay in Vienna. The authors acknowledge financial support of the ESI, the DFG network Grant Oe 538/3-1, OeAD (PL 02/2013) and MNiSW (AT 2/2013-15). Boronski's work was supported by the European Regional Development Fund in the IT4Innovations Center of Excellence Project (CZ.1.05/1.1.00/02.0070). Boroński also gratefully acknowledges the partial support from the MSK DT1 Support of Science and Research in the Moravian-Silesian Region 2013 (RRC/05/2013). The research of Oprocha was supported by the Polish Ministry of Science and Higher Education from sources for science in the years 2013-2014, Grant no. IP2012 004272.

Open Access This article is distributed under the terms of the Creative Commons Attribution 4.0 International License (http://creativecommons.org/licenses/by/4.0/), which permits unrestricted use, distribution, and reproduction in any medium, provided you give appropriate credit to the original author(s) and the source, provide a link to the Creative Commons license, and indicate if changes were made.

\section{References}

1. Alsedá, L., Libre, J.L., Misiurewicz, M.: Combinatorial Dynamics and Entropy in Dimension One, Advanced Series in Nonlinear Dynamics 5. World Scientific, Singapore (1993)

2. Alsedà, L., del Río, M.A., Rodríguez, J.A.: Transitivity and dense periodicity for graph maps. J. Differ. Equ. Appl. 9, 577-598 (2003)

3. Auslander, J., Katznelson, Y.: Continuous maps of the circle without periodic points. Israel J. Math. 32(4), 375-381 (1979)

4. Barge, M.: Horseshoe maps and inverse limits. Pacific J. Math. 121(1), 29-39 (1986)

5. Barge, M.: Homoclinic intersections and indecomposability. Proc. Am. Math. Soc. 101, 541-544 (1987)

6. Barge, M.: A method for constructing attractors. Ergodic Theory Dyn. Syst. 8(3), 331-349 (1988)

7. Barge, M., Martin, J.: The construction of global attractors. Proc. Am. Math. Soc. 110(2), 523-525 (1990)

8. Barge, M., Diamond, B.: The dynamics of continuous maps of finite graphs through inverse limits. Trans. Am. Math. Soc. 344(2), 773-790 (1994)

9. Barge, M., Holte, S.: Nearly one-dimensional Hénon attractors and inverse limits. Nonlinearity 8(1), 29-42 (1995)

10. Bellamy, D.P., Lewis, W.: An orientation reversing homeomorphism of the plane with invariant pseudoarc. Proc. Am. Math. Soc. 114(4), 1145-1149 (1992)

11. Bing, R.H.: Concerning hereditarily indecomposable continua. Pacific J. Math. 1, 43-51 (1951)

12. Blanchard, F.: A disjointness theorem involving topological entropy. Bull. S.M.F. 121, $465-478$ (1993)

13. Blanchard, F., Huang, W.: Entropy sets, weakly mixing sets and entropy capacity. Discret. Contin. Dyn. Syst. 20, 275-311 (2008)

14. Block, L.: Diffeomorphisms obtained from endomorphisms. Trans. Am. Math. Soc. 214, 403-413 (1975)

15. Block, L.S., Coppel, W.A.: Lecture Notes in Mathematics. Dynamics in one dimension, vol. 1513. Springer, Berlin (1992)

16. Block, L., Keesling, J., Uspenskij, V.V.: Inverse limits which are the pseudoarc. Houston J. Math. 26, 629-638 (2000) 
17. Boroński, J.P., Oprocha, P.: Rotational chaos and strange attractors on the 2-torus. Math. Z. 279, 689-702 (2015)

18. Brown, M.: On the inverse limit of Euclidean $N$-Spheres. Trans. Am. Math. Soc. 96, 129-134 (1960)

19. Bruin, H.: Planar embeddings of inverse limit spaces of unimodal maps. Topology Appl. 96(3), 191-208 (1999)

20. Chen, L., Li, S.H.: Lecture Notes in Pure and Applied Mathematics. Dynamical connections between a continuous map and its inverse limit space. Continuum theory and dynamical systems, vol. 149, pp. 89-97. Dekker, New York (1993)

21. Chéritat, M.: Relatively compact Siegel disks with non-locally connected boundaries. Math. Ann. 349(3), 529-542 (2011)

22. Dou, D., Ye, X., Zhang, G.: Entropy sequence and maximal entropy sets. Nonlinearity 19, 53-74 (2006)

23. Efremova, L.S.: Periodic orbits and the degree of a continuous map of a circle (in Russian). Diff. Integer. Equ. (Gor'kii) 2, 109-115 (1978)

24. Fearnley, L.: Characterizations of the continuous images of the pseudo-arc. Trans. Am. Math. Soc. 111, 380-399 (1964)

25. Fearnley, L.: Classification of all hereditarily indecomposable circularly chainable continua. Trans. Am. Math. Soc. 168, 387-401 (1972)

26. Handel, M.: A pathological area preserving $C^{\infty}$ diffeomorphism of the plane. Proc. Am. Math. Soc. 86, 163-168 (1982)

27. Henderson, G.W.: The pseudo-arc as an inverse limit with one binding map. Duke Math. J. 31, 421-425 (1964)

28. Herman, M.: Construction of some curious diffeomorphisms of the Riemann sphere. J. Lond. Math. Soc. 34(2), 375-384 (1986)

29. Huang, W., Ye, X.: A local variational relation and applications. Israel J. Math. 151, 237-280 (2006)

30. Ingram, W.T., Mahavier, W.S.: Inverse Limits. From Continua to Chaos. Developments in mathematics, vol. 25. Springer, New York (2012)

31. Ito, S.: An estimate from above for the entropy and the topological entropy of a $C^{1}$-diffeomorphism. Proc. Jpn. Acad. 46, 226-230 (1970)

32. Kawamura, K., Tuncali, M., Tymchatyn, E.D.: Hereditarily indecomposable inverse limits of graphs. Fund. Math. 185, 195-210 (2005)

33. Kennedy, J., Yorke, J.A.: Pseudocircles in dynamical systems. Trans. Am. Math. Soc. 343, 349-366 (1994)

34. Kennedy, J., Yorke, J.A.: Bizarre topology is natural in dynamical systems. Bull. Am. Math. Soc. (N.S.) 32, 309-316 (1995)

35. Kerr, D., Li, H.: Independence in topological and $C^{*}$-dynamics. Math. Ann. 338, 869-926 (2007)

36. Llibre, J., Misiurewicz, M.: Horseshoes, entropy and periods for graph maps. Topology 32, 649-664 (1993)

37. Minc, P., Transue, W.R.R.: A transitive map on $[0,1]$ whose inverse limit is the pseudoarc. Proc. Am. Math. Soc. 111(4), 1165-1170 (1991)

38. Misiurewicz, M.: Embedding inverse limits of interval maps as attractors. Fund. Math. 125(1), 23-40 (1985)

39. Miyazawa, M.: Chaos and entropy for circle maps. Tokyo J. Math. 25(2), 453-458 (2002)

40. Moise, E.E.: Graduate Texts in Mathematics. Geometric topology in dimensions 2 and 3, vol. 47. Springer, New York (1977)

41. Mouron, C.: A chainable continuum that admits a homeomorphism with entropy of arbitrary value. Houston J. Math. 35(4), 1079-1090 (2009)

42. Mouron, C.: Entropy of shift maps of the pseudo-arc. Topology Appl. 159, 34-39 (2012)

43. Szczechla, W.: Inverse limits of certain interval mappings as attractors in two dimensions. Fund. Math. 133(1), 1-23 (1989)

44. Oversteegen, L., Tymchatyn, E.: On hereditarily indecomposable compacta. Geometric and Algebraic Topology, vol. 18, pp. 407-417. Banach Center Publications, Warsaw (1986)

45. Rogers Jr, J.T.: Pseudo-circles and universal circularly chainable continua. Ill. J. Math. 14, 222-237 (1970)

46. Rogers Jr, J.T.: Mapping the pseudo-arc onto circle-like, self-entwined continua. Mich. Math. J. 17, 91-96 (1970)

47. Walters, P.: An Introduction to Ergodic Theory. Springer, Berlin (2001)

48. Williams, R.F.: One-dimensional non-wandering sets. Topology 6, 473-487 (1967) 